\title{
ACOMPANHAMENTO NUTRICIONAL ASSOCIADO AO TREINAMENTO RESISTIDO PROGRESSIVO SOBRE A ADIPOSIDADE EM HOMENS COM SOBREPESO E OBESIDADE
}

\author{
Nutritional follow-up associated with progressive resistance training on \\ adiposity in overweight and obese men
}

\begin{abstract}
Victoria Pereira Leite Costa ${ }^{1}$; Eduarda Carolina Balena Schinaider²; Vivian Polachini Skzypek Zanardo $^{3 ;}$ Gabriela Pegoraro Zemolin ${ }^{4}$; Roseana Baggio Spinelli5; Gisele Meneghetti ${ }^{6}$; Janesca Mansur Guedes ${ }^{7}$; Mari Lúcia Sbardelotto ${ }^{8}$
\end{abstract}

\begin{abstract}
${ }^{1}$ Acadêmica do Curso de Nutrição da Universidade Regional Integrada dos Alto Uruguai e das Missões; URI Erechim, RS.E-mail: victoriaaplcosta@gmail.com

${ }^{2}$ Nutricionista, Diplomada pela Universidade Regional Integrada do Alto Uruguai e das Missões - URI Erechim, RS

${ }^{3}$ Nutricionista, Docente do Curso de Nutrição da Universidade Regional Integrada do Alto Uruguai e das Missões, URI Erechim, RS, Doutora em Gerontologia Biomédica (PUCRS)

${ }^{4}$ Nutricionista' Docente do Curso de Nutrição da Universidade Regional Integrada do Alto Uruguai e das Missões, URI Erechim, RS, Mestre em Engenharia de Alimentos (URI)

${ }^{5}$ Nutricionista, Docente do Curso de Nutrição da Universidade Regional Integrada do Alto Uruguai e das Missões, URI Erechim, RS, Mestre em Gerontologia Biomédica (PUCRS)

${ }^{6}$ Acadêmica do Curso de Fisioterapia da Universidade Regional Integrada dos Alto Uruguai e das Missões; URI Erechim, RS

${ }^{7}$ Fisioterapeuta, Docente do Curso de Fisioterapia da Universidade Regional Integrada do Alto Uruguai e das Missões, URI Erechim, Doutora em Ciências da Saúde (UNESC)

${ }^{8}$ Educadora Física, Docente do Curso de Educação Física da Universidade Regional Integrada do Alto Uruguai e das Missões, URI Erechim, RS, Doutora em Fisiologia e Bioquímica do Exercício (UNESC)
\end{abstract}

Data do recebimento: 27/10/2020 - Data do aceite: 02/02/2021

RESUMO: Sobrepeso e obesidade representam um problema de saúde mundial, sendo definidos como acúmulo anormal ou excessivo de gordura que pode oferecer riscos à saúde, sua etiologia depende de diversos fatores, incluindo consumo alimentar e atividade física. O objetivo deste estudo foi 
avaliar os efeitos da associação entre a dieta e o treinamento resistido progressivo, em homens adultos com excesso de peso. Pesquisa longitudinal, quase-experimental, caráter quantitativo. Completaram o estudo 27 indivíduos, sexo masculino, idade entre 24 e 57 anos, distribuídos em 4 grupos: Intervenção Nutricional (GIN, n=9), Atividade Física (n=6), Intervenção Nutricional e Atividade Física (GINAF, $n=6)$, Controle $(n=6)$. A maioria dos participantes apresentou redução de Índice de Massa Corporal, passando da classificação de obesidade grau I para sobrepeso, e houve redução do risco de doenças associadas à obesidade para Circunferência da Cintura. Observou-se uma diminuição na média do Valor Energético Total para todos os grupos. O consumo de carboidratos mostrou-se inferior ao recomendado, gramas de proteína/kg de peso adequadas, e lipídeos diminuição na ingestão, exceto para grupo controle. As intervenções promoveram melhoras em parâmetros antropométricos e alimentares, principalmente no GIN e GINAF, apresentando adequação do estado nutricional, podendo colaborar para a promoção da saúde, qualidade de vida dos participantes.

Palavras-chave: Obesidade. Sobrepeso. Estado Nutricional. Dieta.

ABSTRACT: Overweight and obesity represent a global health problem, it is defined as abnormal or excessive fat accumulation that may cause health risks, its etiology depends on several factors, including food consumption and physical activity. The aim of this study was to evaluate the effects of the association between diet and progressive resistance training in overweight adult men. It is a Longitudinal, quasi-experimental quantitative research. 27 male individuals reached the end of the study, age between 24 and 57 years old, who were distributed in 4 groups: Nutritional Intervention (GIN, $n=$ 9), Physical Activity $(\mathrm{n}=6)$, Nutritional Intervention and Physical Activity (GINAF, $n=6)$, Control $(n=6)$. Most of the participants showed reduction in their Body Mass Index, their classification changed from obesity level I to overweight, and there was a reduction in the risk of diseases associated with obesity to Waist Circumference. There was a decrease in the average Total Energy Value for all groups. The consumption of carbohydrates was lower than recommended, grams of protein / $\mathrm{kg}$ of adequate weight, and lipids decrease in intake, except for the Control group. The interventions promoted improvements in anthropometric and dietary parameters, mainly in the GIN and GINAF groups, presenting nutritional status adequacy, which might collaborate for the promotion of health, and the participants quality of life.

Keywords: Obesity. Overweight. Nutritional Status. Diet. 


\section{Introdução}

Sobrepeso e obesidade são situações em que a massa corporal é superior ao padrão para a estatura, e gordura excessiva generalizada ou localizada, respectivamente. Embora os termos sejam utilizados, muitas vezes, como sinônimos, são tecnicamente diferentes. Enquanto sobrepeso é o aumento exclusivo de massa corporal, a obesidade classifica-se como o excesso de adiposidade em sua composição, comprometendo a saúde da população (DE CARVALHO; DUTRA, 2009).

Segundo a Organização Mundial da Saúde (OMS), um em cada oito adultos em todo o planeta é obeso. A projeção é que, em 2025, cerca de 2,3 bilhões de adultos estejam com sobrepeso; e mais de 700 milhões, obesos (ABESO, 2016).

Com etiologia complexa e multifatorial, a obesidade pode ser atribuída a uma interação entre o ambiente, a predisposição genética e o comportamento humano (KOPELMAN, 2000; ABESO, 2016; NGUYEN; EL-SERAG, 2011). Esse conjunto interfere não apenas na duração e qualidade de vida, mas apresenta implicações diretas na aceitação social dos indivíduos de modo geral (DE CARVALHO; DUTRA, 2009). A obesidade tornou-se o principal problema de saúde pública, levando-se em consideração que a mortalidade por essa causa poderia ser evitada pela sua prevenção (POLSKY et al., 2016; LYSEN; ISRAEL, 2018).

Dados da World Health Organization (WHO) (2015) revelam que o excesso de gordura corporal é um fator de risco, pois está associado a várias doenças crônicas degenerativas não-transmissíveis, como doenças cardiovasculares (DCV), diabetes mellitus Tipo 2 (DM2), hipertensão arterial sistêmica (HAS), osteoartrite e alguns tipos de câncer.
Dados do sistema de Vigilância de Fatores de Risco e Proteção para Doenças Crônicas por Inquérito Telefônico (VIGITEL) de 2020, apontam que no Brasil, a frequência de excesso de peso foi de $55,4 \%$, sendo ligeiramente maior entre os homens $(57,1 \%)$. A frequência dessa condição aumentou com a idade até os 44 anos. Quanto à obesidade, a prevalência de adultos obesos foi de $20,3 \%$, sendo maior entre os homens com idade até os 64 anos. Em relação à frequência da prática de atividade física no tempo livre, foi de $46,7 \%$ entre os homens (BRASIL, 2020).

Visando ao controle e prevenção da obesidade, o exercício físico tem se mostrado como uma ferramenta eficaz, em especial o treinamento aeróbio, e/ou a associação desse com exercícios de força, pois possui benefícios cardiovasculares e está relacionado a um maior gasto de energia, auxiliando, assim, na redução de peso. Para Fleck e Kraemer (2017), o treinamento resistido também conhecido como treinamento de força ou com pesos, tornou-se uma das formas mais populares de exercício para melhorar a aptidão física e condicionamento físico, visto que acaba por exigir uma movimentação da musculatura imposta contra uma resistência. Quando associados às dietas de baixa caloria, promovem uma diminuição da gordura corporal e atenuação das comorbidades geradas pelo excesso de gordura (FONSECA-JUNIOR et al., 2013).

Diante do exposto e da importância de delimitar hábitos mais saudáveis como atividade física e acompanhamento nutricional, o objetivo deste estudo foi avaliar os efeitos da associação entre a dieta e o treinamento resistido progressivo, em homens adultos com excesso de peso.

\section{Material e Métodos}

Estudo longitudinal, exploratório, quase -experimental e de caráter quantitativo, rea- 
lizado de setembro de 2019 a maio de 2020 , com 29 indivíduos, com idade entre 24 e 57 anos, do sexo masculino, classificados segundo IMC, em sobrepeso ou obesidade grau I.

Os critérios para participação da pesquisa voluntária foram: não ter participado de um programa de exercícios resistidos nos últimos 3 meses; não ser tabagista por um período de, no mínimo, 5 anos antes do estudo; não possuir nenhuma doença crônica como arritmias, câncer, diabetes ou hepatite; não realizar acompanhamento nutricional, não ter realizado cirurgia bariátrica e concordar em assinar o Termo de Consentimento Livre e Esclarecido (TCLE).

A coleta de informações seguiu o procedimento conforme Figura 1, e ocorreu em um Ambulatório de Especialidades em Nutrição de uma Universidade Comunitária, e uma academia localizada no norte do estado do Rio Grande do Sul. O período de intervenção de cada participante foi de 12 semanas.

$\mathrm{O}$ instrumento de pesquisa foi constituído de três partes. A primeira representada por dados sociodemográficos (faixa etária e estado civil); a segunda referente à avaliação nutricional (consumo alimentar e antropometria); e a terceira pela análise de exames bioquímicos laboratoriais.

Para a coleta de dados foi aplicada a Anamnese Nutricional, que analisou dados clínicos, revisão de sistemas, história familiar e condições e hábitos de vida dos pacientes. Além disso, o padrão alimentar foi averiguado através do Recordatório de 24 horas $(\mathrm{R} 24 \mathrm{H})$, a partir da entrevista individualizada nas terças e quintas-feiras, a fim de descrever e analisar os alimentos e líquidos ingeridos nas últimas 24 horas pelo paciente (FISBERG, 2005). Os dados obtidos foram calculados pelo software AVANUTRI ${ }^{\circledR}$.

Também foi realizada a avaliação antropométrica, segundo KAMIMURA et al., 2019, que contemplou: Peso; Estatura; IMC,
Circunferências da Cintura (CC), Pescoço (CP), Braço (CB), Abdominal (CA), Quadril (CQ); Dobra Cutânea Tricipital (DCT), Dobra Cutânea Bicipital (DCB), Dobra Cutânea Subescapular(DCSE), Dobra Cutânea Supra -ilíaca (DCSI); e Relação Cintura/Estatura (RCE) (KAMIMURA et al., 2019).

Na Tabela I, é possível observar a classificação do IMC e a categoria de risco, segundo a Organização Mundial da Saúde.

Tabela I - Classificação de sobrepeso e obesidade

\begin{tabular}{c|c|c}
\hline Classificação & $\begin{array}{c}\text { Índice de Massa } \\
\text { Corporal } \\
\left(\mathbf{k g} / \mathbf{m}^{\mathbf{2}}\right)\end{array}$ & Risco \\
\hline Sobrepeso & 25,0 a 29,9 & Aumentado \\
Obesidade Grau I & 30,0 a 34,9 & Moderado \\
Obesidade Grau II & 34,9 a 39,9 & Grave \\
Obesidade Grau III & $\geq 40$ & $\begin{array}{c}\text { Muito } \\
\text { grave }\end{array}$ \\
\hline
\end{tabular}

Fonte: Adaptada de WHO (1995); WHO (1997), citado por MUSSOI, 2017.

Para o cálculo do Percentual de Gordura Corporal foram utilizadas as equações de acordo com Durnin e Womersley (1974). Densidade corporal $(\mathrm{DC})=(\mathrm{A}-\mathrm{B}) \times \log \Sigma$ 4 pregas (DCT, DCB, DCSE, DCSI), em que A e B são coeficientes elaborados de acordo com a idade e o sexo para o cálculo da DC. A partir do valor de DC, a porcentagem de gordura corporal total foi determinada utilizando a fórmula de Siri (1961): Gordura corporal $(\%)=4,95-4,5 \times 100$ DC. O diagnóstico foi realizado por LOHMAN; ROCHE; MARTORELL, (1991).

A avaliação de Bioimpedância, foi efetuada através da balança de bioimpedância elétrica Omron ${ }^{\circledR}$, modelo HBF-514. Os dados fornecidos e utilizados pela bioimpedância nesta pesquisa foram: peso corporal $(\mathrm{kg})$; IMC $\left(\mathrm{kg} / \mathrm{m}^{2}\right)$; gordura corporal total $(\mathrm{kg})$ e gordura visceral $\left(\mathrm{cm}^{2}\right)$. O diagnóstico 
Figura 1 - Fluxograma dos participantes do estudo

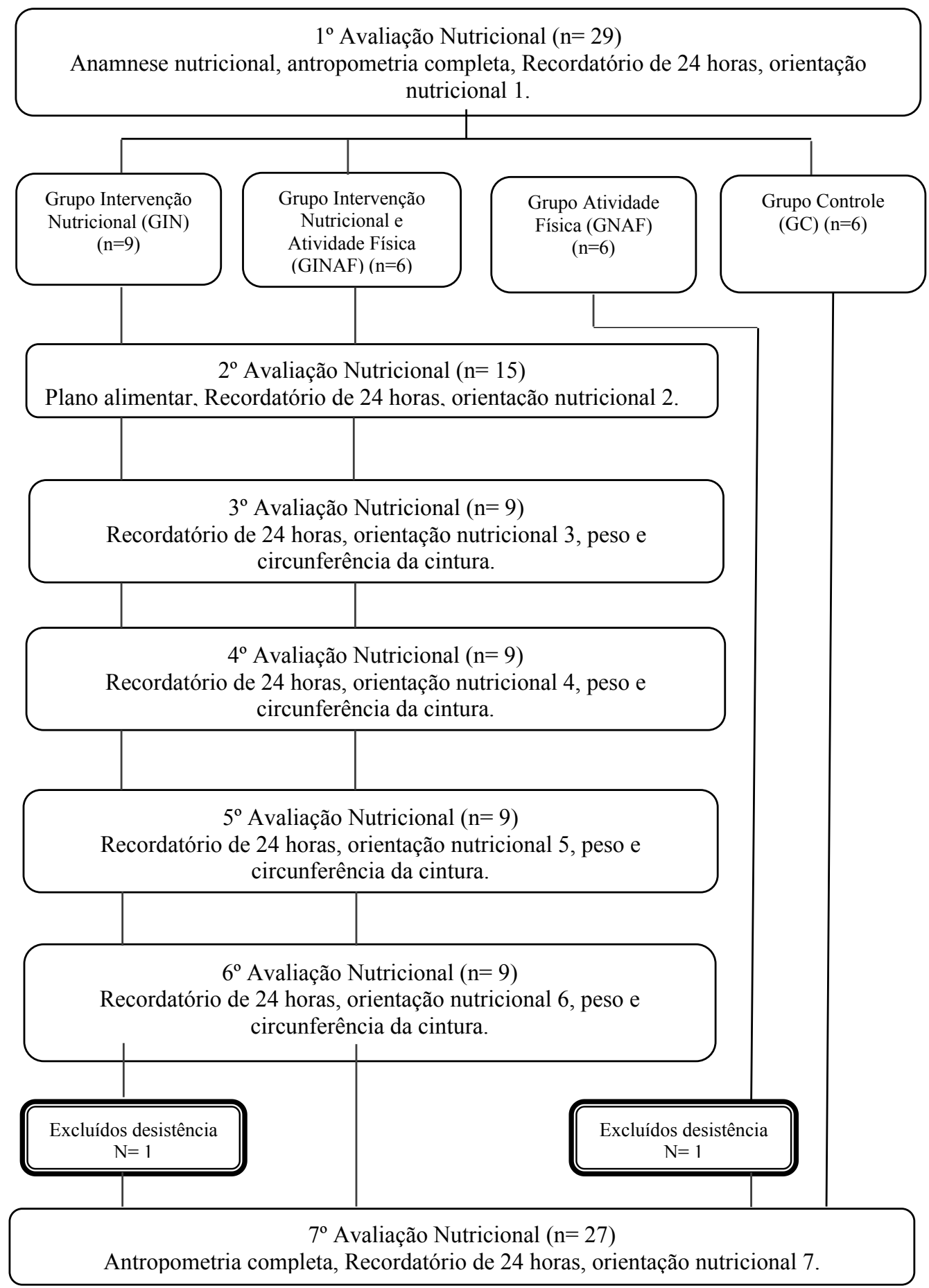


para classificação do percentual de gordura corporal foi através de Jackson e Pollock (1978) de Jackson et al. (1980).

A educação nutricional e alimentar realizada abordou a temática da alimentação saudável, incluindo orientações para sobrepeso, lista de substituição de alimentos, maior consumo de líquidos, ciclo vicioso do açúcar, aprender a reconhecer necessidades como fome, apetite e saciedade, e abordagens de como realizar refeições com atenção plena.

Os dados numéricos obtidos pelos questionários, foram analisados por estatística descritiva com percentual, média e desvio padrão, e foram representados por tabelas.

O projeto foi analisado e aprovado pelo Comitê de Ética em Pesquisa da Universidade Regional Integrada do Alto Uruguai e das Missões - Erechim com parecer $\mathrm{n}^{\circ} 2.953 .460$ e CAAE número 70379817.0.0000.5351.

\section{Resultados e Discussão}

Participaram do estudo 29 indivíduos, do sexo masculino, porém, 2 (GIN n= 1; GNAF $\mathrm{n}=1$ ) desistiram antes da última avaliação. Dessa forma, concluíram a pesquisa 27 homens, com idade entre 24 a 57 anos, e média de 40,8 $\pm 10,6$ anos.

A média de idade encontrada em estudo realizado por Souza et al. (2010), com uma amostra probabilística da população adulta (> 20 anos de idade), residente no Município de Niterói/RJ, foi de 43,0 0 , 7 anos para os homens, exibindo uma população relativamente jovem, com IMC médio de $25,4 \pm 0,2 \mathrm{~kg} / \mathrm{m}^{2}$, corroborando com a média de idade desta pesquisa, o que aponta para um diagnóstico nutricional de risco. Os dados sociodemográficos encontram-se na Tabela II.

Em relação ao estado nutricional dos pacientes, detalhado na Tabela III, é possível analisar de acordo com o IMC, que $37 \%$ $(n=10)$ dos pacientes apresentaram estado nutricional de sobrepeso no início da pesquisa e $63 \%(n=17)$ eram obesos grau I. Ao final da mesma observou-se um aumento na faixa de sobrepeso $(40,7 \%, n=11)$ e uma redução na obesidade Grau I (51,9\%, $\mathrm{n}=14)$.

Tabela II - Distribuição do número e percentual de pacientes participantes da pesquisa por faixa etária e estado civil

\begin{tabular}{lcc}
\hline \multicolumn{1}{c}{ Variáveis } & $\mathbf{N}=\mathbf{2 7}$ & $\mathbf{\%}$ \\
\hline Idade (anos) & 7 & \\
$20-30$ & 4 & 25,93 \\
$31-40$ & 11 & 14,81 \\
$41-50$ & 5 & 40,74 \\
$51-60$ & & 18,52 \\
Estado Civil & 20 & \\
Casado & 7 & 74,07 \\
Solteiro & & 24,93 \\
\hline
\end{tabular}

Através da RCE e CC, verificou-se que em todos os grupos houve prevalência para risco de desenvolvimento de DCV, DM2 e mortalidade por todas as causas, no início do estudo. No entanto, ao final das 12 semanas, ocorreu uma diminuição em ambas as medidas, apontando para redução no risco de complicações associadas ao excesso de peso no GIN (Tabela III).

Segundo Reis et al. (2018), a RCE tem sido proposta como uma alternativa no uso de indicadores antropométricos e está fortemente associada a diversos fatores de risco coronariano, HAS, intolerância à glicose, DM2 e dislipidemias. Além disso, foi observado que os indicadores que fornecem informações de distribuição de gordura na região central - CC e RCE - são superiores no sentido de prever fatores de risco metabólicos em relação ao IMC.

No estudo de Flegal et al. (2008) foram comparados o percentual de gordura corporal (\%GC) com os indicadores antropométricos IMC; CC e RCE em 12.901 adultos, sec- 
cionados por faixa etária. Os autores constataram que em homens, o \% GC foi mais correlacionado com a $\mathrm{CC}$ e $\mathrm{RCE}$, quando comparados ao IMC.

Barbosa et al. (2017) evidenciaram a associação entre o IMC e a $\mathrm{CP}$, além de sua relação com fatores de risco para DCV. Em nosso estudo, observou-se, conforme diagnóstico para $\mathrm{CP}$, que $100 \%$ dos participantes, em todos os grupos, apresentaram valores acima do adequado para os dois momentos da pesquisa, início e final, sugerindo assim excesso de peso por este parâmetro. Além disso, Zanuncio et al. (2017), em seu estudo transversal, também revelaram que pessoas de ambos os sexos com CP acima do ponto de corte para risco cardiometabólico, indicaram maior chance de ocorrência de um evento coronariano em 10 anos.

Em estudo publicado no Journal of the Endocrine Society, no portal Sciencedaily, em 2020, pesquisadores combinaram um programa com dieta restrita em calorias e seis meses de exercício supervisionado. $\mathrm{O}$ estudo, semelhante a este, incluiu 60 adultos com obesidade ou excesso de peso que foram divididos em dois grupos: "condicionamento físico muito ruim" e "condicionamento físico ruim ou melhor". O IMC foi maior naqueles com condicionamento físico muito ruim no início do estudo, em comparação com aqueles do grupo com condicionamento físico ruim ou melhor. Ao final do programa, aqueles na categoria "ruim ou melhor" perderam quase o dobro do peso do que aqueles que tinham uma condição física muito fraca no início do estudo. Contudo, os resultados obtidos nesta pesquisa mostraram que o GIN $(\mathrm{n}=9)$ foi o que apresentou melhores resultados em relação ao IMC e percentual de gordura, indicando maior redução de peso que o restante dos grupos.

Observa-se na Tabela IV a distribuição média do Valor Calórico Total, macronutrien- tes e micronutrientes do GIN, GNAF, GINAF e GC, de acordo com o R24h, realizado na primeira e última avaliação. Analisando o consumo alimentar, observou-se uma diminuição na média do Valor Energético Total (VET), de 1.973,8 1746,0 kcal para $1.776,8 \pm 694,8 \mathrm{kcal}$, sendo esta redução mais considerável no GIN $(2.128,8 \pm 777,1$ kcal para $1.817,2 \pm 404,1 \mathrm{kcal})$ e GINAF $(1.924,8 \pm 649,2 \mathrm{kcal}$ para $1.592,9 \pm 429,6$ kcal).

Em relação aos carboidratos, o consumo mostrou-se inferior ao recomendado pela terapia nutricional para obesidade (de 55 a $60 \%$ do VET) em todos os grupos, enquanto a ingestão de proteínas totais revelou-se levemente aumentada nas avaliações inicial e final conforme valores recomendados (15 a $20 \%$ do VET). Além disso, as gramas de proteína/kg de peso mantiveram-se de acordo com a terapia, entre 0,8 e $1,3 \mathrm{~g} / \mathrm{kg}$ de peso, visto que a mesma preconiza a ingestão de pelo menos $0,8 \mathrm{~g} / \mathrm{kg}$ de peso.

Quanto ao consumo de lipídeos, houve diminuição na ingestão dos mesmos no GIN, GINAF e GNAF entre a primeira e última consultas, enquanto no $\mathrm{GC}$ ocorreu aumento do macronutriente $(25,9 \% \pm 7,0$ para $28,9 \% \pm \mathbf{3 , 6}$ ). Contudo, os valores permaneceram dentro dos parâmetros preconizados pela terapia utilizada para obesidade, de 20 a $30 \%$ do VET.

Em estudo realizado por Rendeiro et al. (2018), em 21 homens obesos, encontraram-se valores similares aos desta pesquisa, em que, para energia, o valor médio foi de $1.937,28 \mathrm{kcal}$. Quanto ao percentual de consumo, obteve-se $51,25 \%$ de ingestão de carboidratos, seguido por $19,94 \%$ de proteínas e $28,31 \%$ de lipídeos.

Lopes et al. (2017) realizaram um programa de intervenção nutricional associado a exercícios físicos de alta intensidade, e observaram que após a intervenção, a inges- 


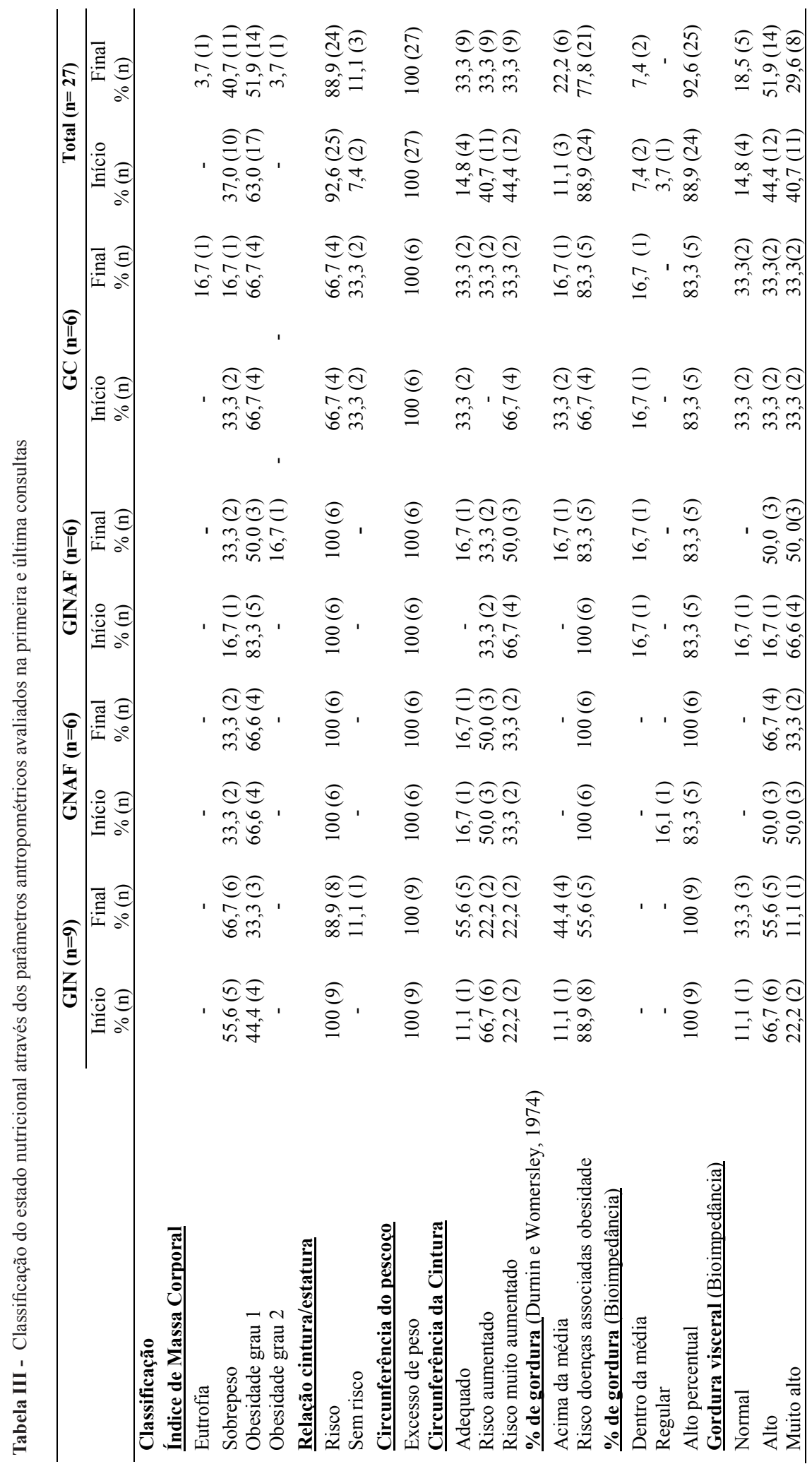




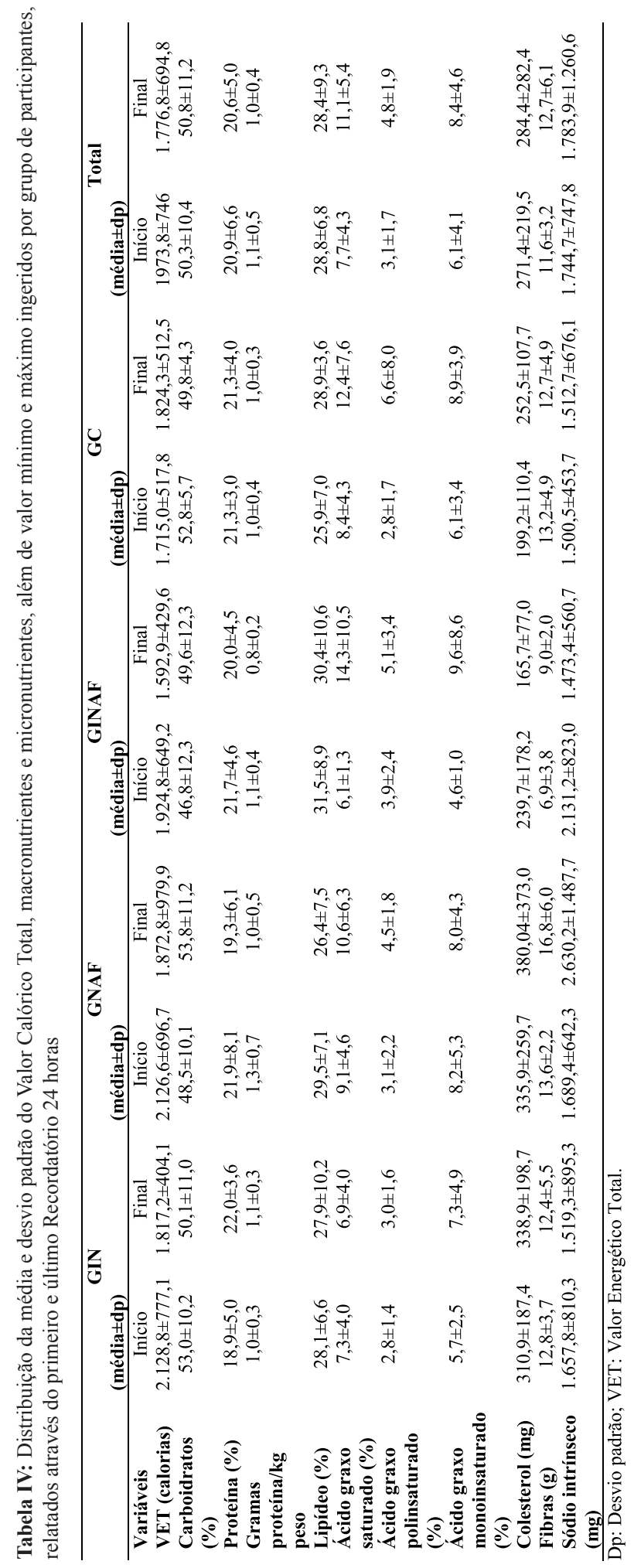


tão de calorias totais diminuiu. Além disso, houve redução de carboidratos e proteínas, e também se observou uma redução do IMC e do percentual de gordura, dados semelhantes ao do presente estudo, em que ocorreu diminuição dos mesmos parâmetros no GINAF.

As fibras alimentares podem reduzir o risco de desenvolvimento de algumas doenças crônicas como DCV, HAS, DM2 e algumas desordens gastrointestinais. Além disso, promovem uma melhora dos níveis dos lipídeos séricos, reduzem os níveis de pressão arterial e melhoram o controle da glicemia em pacientes com DM (BERNAUD; RODRIGUES, 2013). Conforme dados coletados para a pesquisa, a ingestão de fibras totais $(11,6 \pm 3,2$ na primeira avaliação e $12,7 \pm 6,1$ na segunda avaliação) encontrase abaixo da recomendação adequada de $38 \mathrm{~g}$ de fibras ingeridas para homens (DIETARY REFERENCE INTAKES, 2006), em todos os grupos.

Segundo dados da VIGITEL (BRASIL, 2020), a frequência no consumo de cinco ou mais grupos de alimentos ultra processados foi de $18,2 \%$ entre os entrevistados, sendo mais elevada entre os homens, totalizando $21,8 \%$ destes. Neste estudo, embora o consumo de sódio tenha permanecido dentro do valor recomendado, a ingestão de sódio intrínseco aumentou consideravelmente no GIN, de $1.689,4 \pm 642,3 \mathrm{mg}$ para $2.630,2 \pm 1.487,7 \mathrm{mg}$, enquanto no GINAF a média final diminuiu em relação à inicial de $2.131,2 \pm 823,0 \mathrm{mg}$ para $1.473,4 \pm 560,7 \mathrm{mg}$.

Segundo Pontes et al. (2009), a associação entre dieta e exercícios proporciona uma redução de peso mais eficiente durante curtos e longos períodos, comparando à utilização de apenas uma destas intervenções. Diante disso, a combinação promove maior eficiência na mobilização de ácidos graxos e perda de gordura, além de melhorar o perfil lipídico do obeso.
Em estudos realizados recentemente, presentes nas Diretrizes Brasileiras de Obesidade de 2016, foram avaliados a eficácia na redução do peso corporal de dietas, exercícios físicos e programas combinados com, pelo menos, 12 meses de seguimento, realizados em adultos com sobrepeso e obesidade. No total, 8 estudos foram incluídos, com 1.022 participantes. Com 3 a 6 meses, não houve diferença entre programa combinado e só dieta, mas com 12 meses, o programa combinado foi melhor. Já o programa combinado comparado a exercício foi superior com 3 a 6 meses e 12 a 18 meses (ABESO, 2016).

Por fim, a Diretriz Brasileira de Obesidade (2016) orienta que programas combinados de dieta e aumento de atividade física ou exercícios físicos, ou então de dieta isolada, em pacientes que apresentam sobrepeso e obesidade são recomendados preferencialmente ao aumento de atividade física ou exercícios físicos, para redução do peso corporal (ABESO, 2016)

\section{Considerações finais}

O sobrepeso e a obesidade são considerados uma epidemia mundial. Para que haja melhor condição de diagnóstico e acompanhamento nutricional, os principais pontos a se tomar conhecimento são o peso e o percentual de gordura corporal, além de outras medidas antropométricas que mostram as especificidades de cada indivíduo.

A composição corporal e/ou o perfil lipídico dos pacientes pode ser influenciada pelo hábito alimentar. Conforme IMC, pode-se perceber que a maioria dos participantes apresentou redução do índice, passando da classificação de obesidade grau I para a de sobrepeso. Em relação à $\mathrm{CC}$, também houve redução quanto ao risco muito aumentado para doenças associadas à obesidade. Já quanto ao percentual de gordura, pela ava- 
liação das dobras cutâneas, houve redução nos grupos GIN e GINAF, diminuindo assim o risco de doenças associadas à obesidade. Avaliando o consumo alimentar, foi observado diminuição na ingestão alimentar diária, melhor adequação de proteínas e lipídeos, e baixo consumo de carboidratos e fibras.

Diante dos resultados, o presente estudo demonstrou que um programa de intervenção, durante 12 semanas, consistindo em modificações nos hábitos de vida utilizando dieta hipocalórica e/ou uma associação de dieta e exercícios físicos promoveu uma melhora em parâmetros antropométricos e alimentares, principalmente no GIN e GINAF, indicando que a intervenção nutricional e sua associação com o treinamento resistido foi benéfica em ambos os aspectos, apresentando melhora do estado nutricional, podendo colaborar para a promoção da saúde, qualidade de vida e bem-estar dos participantes.

\section{REFERÊNCIAS}

ABESO. Diretrizes Brasileiras de Obesidade 2016. Disponível em: https://abeso.org.br/wpcontent/uploads/2019/12/Diretrizes-Download-Diretrizes-Brasileiras-de-Obesidade-2016.pdf. Acesso em: 25 mar. 2020.

BARBOSA, P. S.; SANTOS, R. P; MENDONÇA, J. L. S; ROCHA, V. S. Circunferência do pescoço e sua associação com parâmetros antropométricos de adiposidade corporal em adultos: Circumference of the neck and its association with anthropometric parameters of body adiposity in adults. Braspen Journal, v. 32, n. 4, p. 315-320, jul/2017. Disponível em: http://arquivos.braspen. org/journal/out-dez-2017/04-Circunferencia-do-pescoco.pdf. Acesso em: 13 maio 2020.

BERNAUD, F. S. R.; RODRIGUES, T. C. Fibra alimentar: ingestão adequada e efeitos sobre a saúde do metabolismo. Arquivos Brasileiros de Endocrinologia e Metabolismo, n. 6, v. 57, p. 397-405, ago. 2013. Disponível em: http://www.scielo.br/scielo.php?script=sci_arttext\&pid=S000427302013000600001\&lng=en\&nrm=iso. Acesso em: 11 maio 2020.

BRASIL. Instituto Brasileiro de Geografia e Estatística (IBGE). Pesquisa de orçamentos familiares 2008-2009: Análise do consumo alimentar pessoal no Brasil / IBGE, Coordenação de Trabalho e Rendimento. - Rio de Janeiro: IBGE, 2011.

BRASIL. Ministério da Saúde. Secretaria de Vigilância em Saúde. Departamento de Vigilância de Doenças e Agravos não Transmissíveis e Promoção da Saúde. Vigitel Brasil 2020: vigilância de fatores de risco e proteção para doenças crônicas por inquérito telefônico: estimativas sobre frequência e distribuição sociodemográfica de fatores de risco e proteção para doenças crônicas nas capitais dos 26 estados brasileiros e no Distrito Federal em 2019 / Ministério da Saúde, Secretaria de Vigilância em Saúde, Departamento de Vigilância de Doenças e Agravos não Transmissíveis e Promoção da Saúde. - Brasília: Ministério da Saúde, 2020.

DURNIN J. V. G. A.; WOMERSLEY, I. Body fat assessed from total body density ad its estimation from skinfold thickness: measurement on 481 men and women aged from 16 to 72 years. Braspen Journal, v. 32, p.77-97, 1974.

DUTRA, E.S.; de CARVALHO, K.M. Syndrome in central Brazil: prevalence and correlates in the adult population. Diabetol Metab Syndr 2012; 4:20. In: CUPPARI, L. Nutrição Clínica no Adulto. 4. ed. Barueri/SP: Manole, p.186-214, 2019, 
FISBER, M. R.; SLATER, B; MARCHIONI, D. M. L; MARTINI, L. A. Inquéritos Alimentares: Métodos e bases científicos. Barueri, SP: Manole, 2005.

FLEGAL, K. M.; SHEPHERD, A. C. L; GRAUBARD, B. I; BORRUD, L. G; OGDEN, C. L; HARRIS, T. B; EVERHART, J. E; SCHENKER, N. Comparisons of percentage body fat, body mass index, waist circumference, and waist-stature ratio in adults. The American Journal of Clinical Nutrition, v. 89, n. 2, p. 500-508, dez/2008. Disponível em: https://academic.oup.com/ajcn/ article/89/2/500/4596685. Acesso em: 25 maio 2020.

FONSECA-JUNIOR, D. J.; BUSTAMANTE SÁ, C. G. A; RODRIGUES, P. A. F; OLIVEIRA, A. J; FERNANDES-FILHO, J. Exercício físico e obesidade mórbida: uma revisão sistemática. ABCD, Arquivos brasileiros de cirurgia digestiva, v. 26, supl. 1, p. 67-73, 2013. Disponível em: http:// www.scielo.br/scielo.php?script=sci_arttext\&pid=S010267202013000600015\&lng=en\&nrm=iso. Acesso em: 28 maio 2020.

JACKSON, A.S.; POLLOCK, M.L.; WARD, A. Generalized equations for predicting body density of women. Medicine \& Science in Sports \& Exercise, v. 12, p.175-82, 1980

KOPELMAN, P.G. Obesity as a medical problem. Nature, v. 404, n. 6778, p. 635643, 2000.

LOHMAN T. G.; ROCHE, A. F.; MARTORELL, R. Anthropometric standardization reference manual. Abridged, 1991. p.90

LOPES, J.; MATOS, M. A; MAGALHÃES, F. C; ESTEVES, E. A; VIEIRA, E. R; AMORIM, F. T. Efeito de mudanças graduais de exercício físico e dieta sobre a composição corporal de obesos. Arquivos de Ciências da Saúde, v. 24, n.1, p. 93-97, 2017. Acesso em: 20 maio 2020.

LYSEN, K, L; ISRAEL. Nutrição no Controle da Massa Corporal. In: KATHLEEN, Mahan L.

Krause Alimentos, Nutrição e Dietoterapia. 14. ed. São Paulo: Elsevier, Cap. 21. p. 546-564, 2018.

MAHAN, L. K.; ESCOTT-STUMP, S.; JANICE, L. Krause, alimentos, nutrição e dietoterapia. 13. ed. Edição Digital. Rio de Janeiro: Elsevier, 2013. SILVA, Sandra Maria Chemin Seabra da; MURA, Joana D'Arc Pereira. Tratado de alimentação, nutrição e dietoterapia. 2. ed. São Paulo: Roca, 2011.

MUSSOI, T.; D. Avaliação Nutricional na Prática Clínica: da gestação ao envelhecimento. 1. ed. Rio de Janeiro: Guanabara Koogan, 2017. p. 65

NGUYEN, D. M.; EL-SERAG, H. B. The epidemiology of obesity. Gastroenterology Clinics of North America, v. 39, n. 1, p. 1-7, 2010.

POLSKY, V. A. S. Obesidade: epidemiologia, etiologia e prevenção. In: ROSS, Catharine et al. (Ed.). Nutrição Moderna de Shils: na Saúde e na Doença. 11. ed. Barueri: Manole, Cap. 58. p. 775$787,2016$.

PONTES, A. L. C; SOUSA, I. A. D; NAVARRO, COPPI, A. O tratamento da obesidade através da combinação dos exercícios físicos e terapia nutricional visando o emagrecimento. Revista Brasileira de Obesidade, Nutrição e Emagrecimento, v. 3, n. 14, p. 124-135, mar/abri 2009. Acesso em: 04 jun. 2020.

REIS, G. M. S; ARAÚJO, S. M; MEDEIROS, J. M. B; MENEZES, A. F. A. Razão cintura/estatura e indicadores antropométricos de adiposidade: Waist-to-height ratio and anthropometric indicators of adiposity. Braspen Journal, v. 33, n. 4, p. 435-439, abr./2018. Disponível em: http://arquivos.braspen. org/journal/out-dez-2018/artigos/12-AO-Razao-Cintura-estatura.pdf. Acesso em: 25 maio 2020.

RENDEIRO, L. C; FERREIRA, C. R; SOUZA, A. A. R; OLIVEIRA, D. A; DELLA NOCE, R. R. Consumo alimentar e adequação nutricional de adultos com obesidade. Revista Brasileira de Obesidade, Nutrição e Emagrecimento, v. 12, n. 76, p. 996-1008, jan/dez 2018. Disponível em: http://www.rbone.com.br/index.php/rbone/article/view/837/620. Acesso em: 18 maio 2020. 
SCIENCEDAILY. Poor fitness may impede long-term success in weight loss program.

Disponível em: https://www.sciencedaily.com/releases/2020/03/ 200331130033. html. Acesso em: 28 maio 2020.

SOUZA, D. R; DOS ANJOS, L. A; WAHRLICH, V; VASCONCELLOS, M. T. L; MACHADO, J. M. Ingestão alimentar e balanço energético da população adulta de Niterói, Rio de Janeiro, Brasil: resultados da Pesquisa de Nutrição, Atividade Física e Saúde (PNAFS). Caderno. Saúde Pública, v. 26, n. 5, p. 879-890, Mai 2010. Disponível em: http://www.scielo.br/scielo.php?script=sci arttext\&pid=S0102-311X2010000500010\&lng=en\&nrm=iso. Acesso em: 03 jun. 2020. https://doi. org/10.1590/S0102-311X2010000500010.

WORLD HEALTH ORGANIZATION. World Health Statistics 2015. Disponível em: https://www. who.int/gho/publications/world_health_statistics/EN_WHS2015_Part2.pdf?ua=. Acesso em: 25 mar. 2020

ZANUNCIO, V. V; PESSOA, M. C; PEREIRA, P. F; LONGO, G. Z. Neck circumference, cardiometabolic risk, and Framingham risk score: Population-based study. Revista de Nutrição, v. 30, n. 6, p. 771-781, 2017. 
